\title{
La planification du développement dans les petites collectivités : quelques réflexions
}

\author{
Lucie Houle et Paul Prévost ${ }^{1}$ \\ Institut de recherche et d'enseignement pour les coopératives \\ de l'Université de Sherbrooke (IRECUS)
}

\section{Introduction}

Le développement et le dynamisme des collectivités rurales varient substantiellement de l'une à l'autre. Certaines collectivités rurales, plus prospères et plus dynamiques, réussissent à s'ajuster à leur environnement et défient les tendances défavorables qui nuisent à leur développement. D'autres, sans réel dynamisme et capacité d'adaptation, connaissent une diminution de leur croissance, un dépeuplement, un désintérêt pour la vie communautaire, une perte de sentiment d'appartenance de la population, etc.

Plusieurs facteurs influencent le développement des collectivités rurales. Les caractéristiques géographiques, les choix politiques, l'exode, le vieillissement de la population et bien d'autres. Mais pourtant, il arrive de constater que certaines, même aux prises avec des difficultés importantes, réussissent à relativement bien tirer leur épingle du jeu et à se développer.

Des réflexions, tirées d'interventions réalisées dans des milieux ruraux, tendent à mettre en évidence que le succès d'un projet de développement peut s'expliquer par trois facteurs:

- Le projet est porté par la communauté;

- Le parcours favorise un dénouement cohérent et complet du projet;

- Les porteurs de développement ont les compétences requises pour réaliser le projet.

Le présent article présente chacune de ces trois dimensions. D'entrée de jeu, nous définissons un projet de développement puis expliquons les processus du parcours de développement d'un projet. Dans les deux cas, nous avons illustré nos propos par des interventions réalisées dans des milieux ruraux. Nous mettons ensuite l'accent sur les compétences requises chez les porteurs de développement en nous appuyant sur les résultats et les responsabilités qui leur sont confiés lorsqu'ils ont à franchir un parcours de développement.

Ce texte se veut un outil de réflexion destiné aux praticiens et praticiennes du développement local qui souhaitent s'aventurer dans un projet. Nous espérons qu'il permettra de mieux comprendre le parcours de développement et de cerner les compétences requises pour sélectionner, former et évaluer les porteurs de développement. Aucune prétention de généralisation n'est ici envisagée.

\section{Ce texte se veut un outil de réflexion destiné aux praticiens et praticiennes du développement local qui souhaitent s'aventurer dans un projet.}

\section{Le projet est porté par la communauté}

Le développement d'une collectivité se fait à travers des initiatives, grandes et petites, qui prennent la forme de projets. Ces projets, issus du milieu, peuvent concerner le sentiment d'appartenance à la collectivité, la culture locale, le partenariat, la participation communautaire, l'entrepreneurship, la création d'emplois et autres.

Non seulement le projet est-il issu du milieu, il est aussi porté par le milieu. Ce qui signifie que des personnes mènent le projet, le défendent, lui trouvent un réseau d'appuis, l'évaluent, etc. D'ailleurs, qui est le mieux placé pour bien connaître la réalité et les besoins du milieu, que le milieu lui-même. 
Un projet de développement est global, c'est-à-dire qu'il concerne et implique l'ensemble de la communauté et touche plusieurs secteurs d'activités. Il exige alors un consensus social et favorise une meilleure cohésion. Un projet peut aussi être spécifique. Dans ce cas, il est piloté par des entreprises, des organismes, des individus et concerne un secteur spécifique. Il doit cependant contribuer à la réalisation du projet global de la communauté.

L'identification des responsables et des partenaires, des moyens pour réaliser le projet, des résultats attendus, des indicateurs de résultats et des échéanciers sont toutes des composantes importantes à déterminer pour structurer et actualiser le projet.

\section{Exemple de projet global de développement Élaboration du plan stratégique de la Ville de Stanstead 2}

Responsable: Comité de développement de la Ville de Stanstead

Partenaires: CLE, CLD, CLSC, écoles de la municipalité, Association des gens d'affaires, université, etc.

Les moyens

- L'appropriation d'un cadre de référence par les porteurs de développement;

- La planification et la réalisation de journées de réflexion publiques (sommet des leaders locaux, forum public);

- L'implication des leaders locaux et de la population;

- La mise en place de la structure de suivi et d'appui.

Les résultats

Immédiats

- La naissance d'une vision partagée à l'égard de la problématique, des enjeux et des axes de développement;

- Un consensus sur des priorités;

- L'émergence d'idées de projets;

- L'identification de porteurs de projets;

- La mise en place d'une structure d'accompagnement et de suivi.

Moyen terme

- La mise en œuvre de projets innovateurs adaptés à la réalité;

- L'accès à des données intersectorielles;

- Des stratégies d'alliances ou de réseaux.

Long terme

- La création d'un milieu propice aux initiatives locales;

- La création d'alliances stratégiques et de partenariat;

- Une capacité accrue de la collectivité à utiliser et à adapter sa main-d'œuvre, etc.

$\underline{\text { Les indicateurs }}$

Immédiats

- Le nombre et la pertinence des projets;

- Les résultats de l'évaluation de la satisfaction des activités publiques;

- La proportion représentative des personnes participantes; 
Moyen terme

- Le nombre d'initiatives locales ou de projets nouveaux réalisés;

- Le nombre de plans d'affaires conçus;

- La création de sites Web, de banques de données, de rapports, etc.

- L'activité des comités mis en place.

Long terme

- Le nombre d'initiatives locales ou de projets nouveaux;

- Les résultats d'évaluation des retombées des projets;

- Le nombre d'emplois créés et d'employés formés.

L'échéancier : juin 2002

\section{Exemple de quelques idées de projets spécifiques issus d'un projet global Élaboration d'un profil et de stratégies d'emploi dans la municipalité de Weedon ${ }^{3}$}

\section{$\underline{\text { Projet global }}$}

À la suite de la réalisation d'un plan stratégique local, la communauté de Weedon a identifié, parmi les axes de développement privilégiés, la nécessité de développer et de soutenir la vitalité économique. Le projet consistait à élaborer un profil et des stratégies d'emploi.

Responsable: La Chambre de commerce de Weedon

$\underline{\text { Résultats attendus }}$

À court terme

Un portrait statistique des principales caractéristiques des entreprises et de la main-d'œuvre;

L'état des perceptions à l'égard de l'emploi;

Des enjeux à considérer avant d'amorcer une démarche d'animation et de mobilisation;

Des pistes à explorer pour contribuer à la vitalité économique.

À moyen et long terme

La création d'alliances stratégiques et de partenariat;

La création d'emplois.

Quelques idées de projets spécifiques devant permettre le développement et le soutien de la vitalité économique à Weedon

- Afin de développer et de promouvoir un mécanisme local de recrutement de la main-d'œuvre, créer et promouvoir un babillard (réel et virtuel) mis à la disposition des employeurs et de la population;

- Afin de faire connaître à la population les employeurs locaux et le type d'emploi offert, organiser des visites chez les employeurs, un salon annuel de l'emploi, des rencontres à caractère social réunissant employeurs et main-d'œuvre, etc.;

- Afin de mettre en commun des expertises ou des services pour les entreprises, créer un réseau d'experts en comptabilité ou en gestion des ressources humaines (santé et sécurité au travail, formation, planification de carrières, etc.);

- Etc.... 


\section{Le parcours favorise un dénouement cohérent et complet du projet}

Le parcours pour élaborer, gérer et évaluer un projet de développement est complexe et se fait rarement sans heurt. Lorsque l'on invite la population à se doter d'un projet de développement, il est fort probable que des conflits surgiront, des intérêts divergents naîtront, des attentes non comblées créeront des frustrations ou des désistements. Il vaut donc mieux que les praticiens et les praticiennes du développement local sachent dès le départ qu'une somme d'énergie importante doit être consacrée lorsqu'ils ou elles s'engagent dans un tel processus. $\mathrm{Ne}$ pas en être conscient peut conduire à l'échec, en être conscient peut permettre de prévenir de telles éventualités.

\section{Le parcours pour élaborer, gérer et évaluer un projet de développement est complexe et se fait rarement sans heurt.}

Loin de prétendre que ce que nous allons présenter constitue la recette sans faille, nous proposons un parcours de développement composé de 4 processus interdépendants qui doivent être considérés pour favoriser la réussite d'un projet de développement. Ce parcours donne un sens au projet, l'encadre et y intègre les partenaires appropriés 4 .

\section{- Le processus de réflexion}

Le processus de réflexion tend à aider la communauté à comprendre ce qui se passe et ainsi à mieux agir sur son environnement. Il permet généralement de tracer une lecture complète des forces et faiblesses, des opportunités et menaces des divers secteurs qui composent la communauté, d'y dégager les enjeux, de formuler les axes et les priorités de développement qui marqueront les années subséquentes. En fait, il donne un sens aux actions.

La réflexion est souvent tirée d'activités de formation, de planification, de recherche-action, etc.

\section{- Le processus d'animation}

Le processus d'animation amène la collectivité à s'investir à l'intérieur d'un projet de développement. Différentes formules peuvent être imaginées. Pensons notamment à un sommet, un forum ou un débat public faisant travailler ensemble la communauté à imaginer des solutions, à rêver d'avenir, à innover.

L'animation du milieu peut avoir un impact favorable sur le sentiment d'appartenance et le goût de s'investir. Cette dimension exige généralement une bonne dose d'énergie pour planifier et organiser les événements ainsi que des stratégies adéquates pour mobiliser le milieu.

\section{Les 4 processus du parcours de développement local}

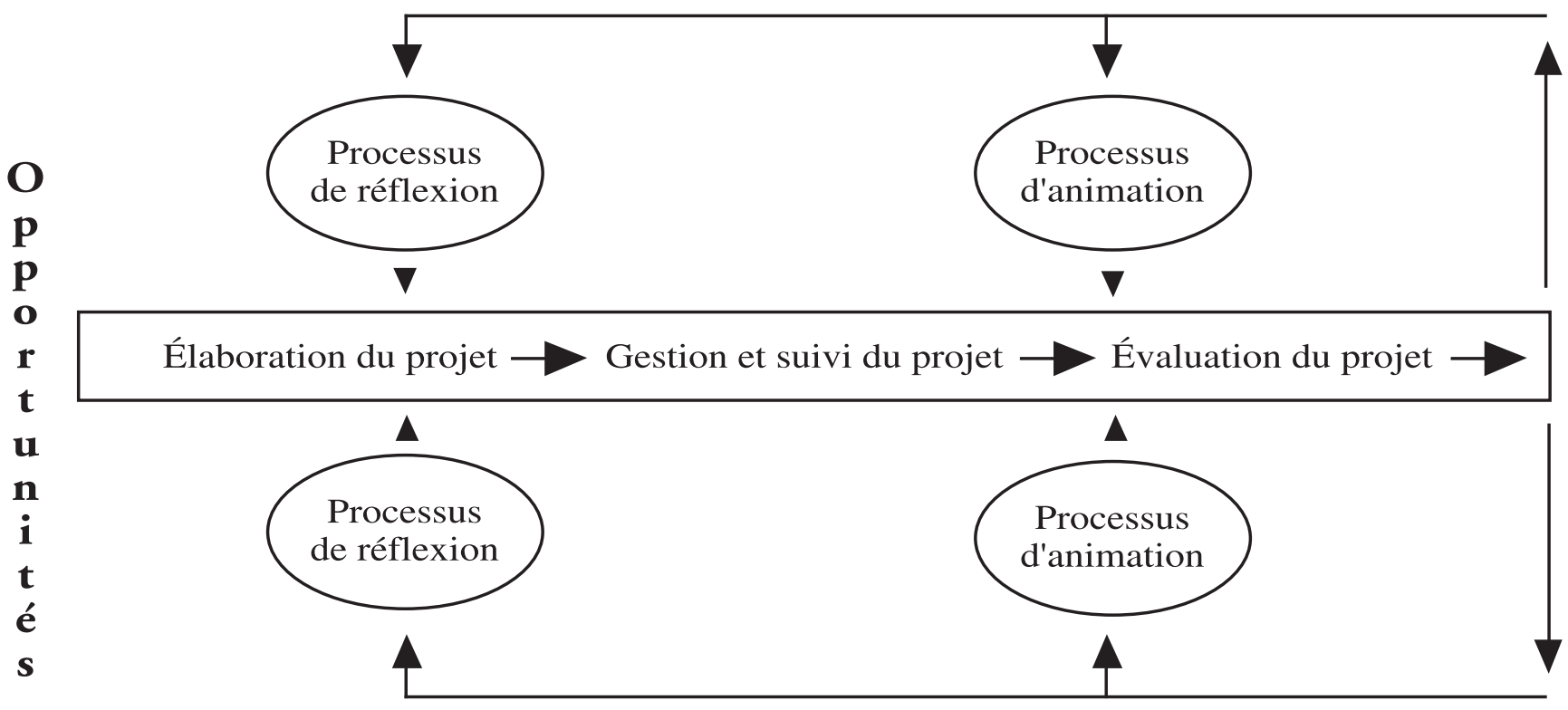




\section{- Le processus d'organisation}

Le processus d'organisation permet de passer du discours à l'action, de se doter d'un réseau d'appuis et d'expertises, d'assurer un support technique, etc. La mise en place de divers mécanismes ou instances de suivi est alors nécessaire. La résultante d'un tel processus devrait être la mise en place d'instances ou de regroupements nouveaux, des mécanismes de prise de décisions ainsi que d'un réseau d'appuis, etc.

\section{- Le processus politique}

Plus intangible que les trois autres dimensions, le processus politique fait référence au pouvoir (capacité de se prendre en main), au leadership (capacité d'influencer) et au partenariat (capacité de se regrouper).

Plusieurs organismes locaux, territoriaux ou régionaux stimulent, orientent et accompagnent le développement local. Le processus politique devrait donner lieu à de nouvelles coalitions ou nouveaux regroupements de partenaires.

Pour mieux illustrer nos propos, l'exemple ci-dessous présente les grandes lignes du travail réalisé par le milieu lors de l'élaboration du plan stratégique de Stanstead et ce, pour chacune des dimensions de la dynamique de développement.

\section{Exemple d'un parcours de développement du projet d'élaboration du plan stratégique pour la ville de Stanstead}

Le processus de réflexion

Une période intense de réflexion a précédé l'exercice de planification stratégique locale. Puisque des porteurs de développement locaux avaient peu d'expériences en matière de planification stratégique, il s'est avéré important que ces personnes soient sensibilisées aux enjeux d'un tel exercice, aux étapes à franchir et puissent aussi se doter d'une vision commune des étapes à accomplir. Les membres des différents comités mis en place pour piloter le projet et les élus municipaux avaient à convenir d'un modèle de planification stratégique et se faire une meilleure idée des enjeux et des étapes à réaliser. Pour favoriser la réflexion, une session de formation et des discussions avec d'autres organismes ayant déjà réalisé un tel exercice ont notamment été organisées.

Le processus d'animation

L'animation du milieu consistait à sensibiliser et à mobiliser la collectivité afin de l'amener à s'investir dans la solution de ses problèmes et à initier un moment propice de développement.

Le comité responsable a convenu d'organiser deux activités publiques. Une première, réunissant les leaders locaux sous la forme d'un sommet et une seconde, réunissant la population de Stanstead sous la forme d'un forum public.

Lors du sommet, les leaders locaux identifiés ont été conviés à :

- Valider et enrichir le portrait des secteurs en mettant en évidence les forces et les faiblesses, les opportunités et les menaces de la municipalité;

- Se donner une vision commune en anticipant et en réfléchissant à l'avenir de Stanstead;

- Identifier les enjeux et les axes de développement en analysant la situation actuelle et souhaitée et en faisant ressortir les orientations.

Lors du forum public, la population de Stanstead a été invitée afin de permettre aux gens de :

- Connaître les résultats du sommet des leaders locaux;

- Identifier des priorités d'action pour les prochaines années; 
- Identifier des priorités d'action pour les prochaines années;

- Formuler des idées de projets;

- S'impliquer dans la réalisation de projet;

- Connaître les suites.

Le processus d'organisation

Pour réaliser un projet de développement, il faut mettre en place un ensemble de moyens crédibles et légitimes et s'appuyer sur les bonnes personnes. La dimension « organisation » est donc cruciale afin d'optimiser les impacts souhaités et assurer la pérennité du projet de développement. Pendant cette période, les membres du comité responsable ont été invités à identifier leur rôle et leur valeur ajoutée, à préciser le rôle et les responsabilités de l'agent de développement et à identifier les étapes et le soutien pour actualiser le plan stratégique.

\section{Le processus politique}

Le comité responsable a identifié les leaders ayant une connaissance des secteurs d'activités et une crédibilité dans le milieu. Ils ont constitué un comité consultatif, formé d'un leader pour chaque secteur. Cette stratégie avait pour intention de se rapprocher des besoins et de la réalité du milieu et d'accéder au réseau de contact de ces personnes.

Les partenaires de l'interne et de l'externe, les élus municipaux de même que les députés provincial et fédéral ont été invités à réfléchir à l'avenir de la municipalité et à s'impliquer.

\section{Les porteurs de développement ont les compétences requises pour réaliser le projet}

Les porteurs de développement ont un rôle prépondérant dans la réussite ou l'échec d'un projet. En effet, le parcours de développement, garni de résistances, d'obstacles et d'une somme considérable de travail, requiert une panoplie de compétences pour ceux qui portent le projet.

\section{Le parcours de développement, garni de résistances, d'obstacles et d'une somme considérable de travail, requiert une panoplie de compétences pour ceux qui portent le projet.}

Le processus de réflexion devrait conduire à l'élaboration d'un plan de développement. Il réclame des capacités à anticiper les tendances, à construire des stratégies, à communiquer, à diriger, etc.
Le processus politique devrait encourager la création d'alliances stratégiques, de regroupements et de nouveaux partenariats. Il requiert des habiletés à négocier, à établir des liens, à cultiver un réseau de contacts, etc.

Le processus d'animation devrait favoriser l'identification de nouveaux leaders de la communauté, des citoyens qui s'impliquent, des projets innovateurs. Il fait appel à des capacités à communiquer sa vision, à rassembler, à repérer les idées nouvelles, etc.

Enfin, le processus d'organisation devrait instiguer la mise en place de mécanismes de suivis et de structures d'appuis. Il sollicite des habiletés à gérer, à organiser et à communiquer ainsi que des aptitudes techniques fonctionnelles.

Pour mieux illustrer notre propos, nous reprendrons, à l'intérieur du tableau ci-dessous, les quatre dimensions de la dynamique de développement et juxtaposerons des exemples de résultats attendus et de responsabilités confiées à des porteurs de développement. Cet exercice nous conduira à l'identification des compétences requises pour chacune des dimensions. 


\section{Les compétences les plus pertinentes pour réaliser un projet de développement ${ }^{5}$}

\begin{tabular}{|c|c|c|c|}
\hline $\begin{array}{l}\text { Dimension du parcours } \\
\text { de développement }\end{array}$ & $\begin{array}{l}\text { Résultats attendus d'un } \\
\text { projet de développement }\end{array}$ & $\begin{array}{l}\text { Responsabilités confiées aux } \\
\text { porteurs de développement }\end{array}$ & Compétences requises \\
\hline Réflexion & $\begin{array}{l}\text { Un plan stratégique } \\
\text { Un plan d'affaires }\end{array}$ & $\begin{array}{l}\text { - Donne un sens aux projets } \\
\text { ou aux actions initiés } \\
\text { - Conçoit des stratégies } \\
\text { gagnantes et originales }\end{array}$ & $\begin{array}{l}\text { Sait concevoir } \\
\text { des stratégies } \\
\text { Sait communiquer } \\
\text { sa vision } \\
\text { Sait diriger } \\
\text { Sait anticiper } \\
\text { les tendances }\end{array}$ \\
\hline Politique & $\begin{array}{l}\text { Des alliances stratégiques } \\
\text { Des regroupements de } \\
\text { citoyens et de partenaires }\end{array}$ & $\begin{array}{l}\text { - Construit et facilite les } \\
\text { coalitions nécessaires } \\
\text { pour appuyer ou piloter } \\
\text { les bonnes idées }\end{array}$ & $\begin{array}{l}\text { Sait établir des liens } \\
\text { de collaboration } \\
\text { Sait négocier } \\
\text { Sait agir habilement } \\
\text { et efficacement }\end{array}$ \\
\hline Organisation & $\begin{array}{l}\text { Des mécanismes de suivi } \\
\text { et des structures d'appui } \\
\text { mis en place } \\
\text { L'accès à un réseau } \\
\text { de support technique }\end{array}$ & $\begin{array}{l}\text { - Met en ouvre le processus } \\
\text { d'organisation pour arriver } \\
\text { à des résultats } \\
\text { - Mobilise les ressources } \\
\text { nécessaires pour faire } \\
\text { avancer les dossiers } \\
\text { - Fixe des objectifs, } \\
\text { des résultats }\end{array}$ & $\begin{array}{l}\text { Sait comment organiser } \\
\text { le travail en fonction des } \\
\text { ressources disponibles } \\
\text { Sait s'adapter } \\
\text { Sait communiquer } \\
\text { l'avancement des travaux } \\
\text { Possède des aptitudes } \\
\text { techniques fonctionnelles }\end{array}$ \\
\hline
\end{tabular}

\section{Conclusion}

Nous constatons que la dynamique de développement d'un projet dans une collectivité exige que les porteurs de développement connaissent le meilleur parcours à franchir pour le réaliser ainsi qu'une vaste étendue de compétences.

Il peut sembler illusoire de rassembler chez une même personne l'ensemble de ces compétences. Les praticiens et les praticiennes qui travaillent dans le domaine du développement local savent bien que dans des petites collectivités, les ressources sont insuffisantes, faute de budget ou de difficultés de recrutement. Il peut donc sembler illusoire de trouver cette perle rare qui sera en mesure de s'acquitter adéquatement de ses responsabilités et atteindre les résultats escomptés.

C'est pour cette raison que tout au long de notre texte nous avons intentionnellement utilisé le pluriel lorsque nous traitions de porteurs de développement. Il est important de miser sur les compétences collectives d'un 
groupe de porteurs de développement plutôt que sur les compétences individuelles d'une seule personne. En effet, le développement local ne doit pas reposer uniquement sur un seul porteur de développement, mais plutôt sur des personnes qui mettront en commun plusieurs compétences et feront fructifier leur portefeuille de compétences.

\section{Il est important de miser sur les compétences collectives d'un groupe de porteurs de développement plutôt que sur les compétences individuelles d'une seule personne.}

Nous pensons également que cette mise en commun de compétences est non seulement requise pour s'acquitter de leurs responsabilités mais aussi pour faire face à d'autres réalités. La complexité croissante des problèmes, la rareté de la main-d'œuvre, l'insuffisance de ressources qualifiées, la fragile stabilité des ressources sont tous des aspects qui militent en faveur de la multiplication des compétences. Qui n'a jamais constaté l'effondrement de projets à la suite du départ d'une personne, un climat tendu par des problèmes non résolus en raison de leur complexité, un porteur de développement démotivé par des résultats non atteints causés par une somme de travail trop importante à réaliser.

Par ailleurs, un groupe soucieux de son développement visera à établir un maillage efficace de compétences non seulement entre les membres de ce groupe mais aussi entre des partenaires externes, des organismes régionaux ou territoriaux, les élus des différents paliers gouvernementaux, le milieu universitaire, etc.

En d'autres mots, les porteurs de développement doivent avoir une compétence incontournable:

\section{«Savoir bien s'entourer»}

\section{Notes et références}

1 Paul Prévost est détenteur d'un doctorat en développement régional de L'Université de Lancaster. Il est professeur au département de management de l'Université de Sherbrooke, directeur du Centre d'excellence en gestion du développement local de la Faculté d'administration de l'Université de Sherbrooke et titulaire de la Chaire Desjardins de coopération et de développement du milieu. Lucie Houle est détentrice d'une maîtrise en éducation à l'Université de Sherbrooke. Gestionnaire de projets, elle a accompagné les villes de Stanstead et de Weedon dans la réalisation de leur projet de développement au cours de l'année 2002. Elle a œuvré plusieurs années dans le domaine du développement des compétences et de la planification de la relève de gestionnaires. Elle est actuellement étudiante au doctorat en administration (DBA) à l'Université de Sherbrooke.

2 L'Institut de recherche et d'enseignement pour les coopératives de l'Université de Sherbrooke (IRECUS) a été mandaté pour accompagner un exercice de planification stratégique pour la Ville de Stanstead. Nous remercions M. Harvey Stevens, agent au Bureau de développement de Stanstead ainsi que les membres du comité de développement.

3 L'Institut de recherche et d'enseignement pour les coopératives de l'Université de Sherbrooke (IRECUS) a été mandaté pour définir un profil et des stratégies d'emploi en concertation avec le milieu. Nous remercions M. Paul Monette, agent de développement à la Chambre de commerce de Weedon ainsi que les membres du comité consultatif.

4 Prévost, Paul ( 2001). Cahiers de recherche IREC 01-05. Les dimensions et processus du développement des collectivités locales. Collection cahiers de recherche. Université de Sherbrooke.

5 L'enchaînement entre les résultats attendus, les responsabilités confiées et les compétences requises a été inspiré par les travaux de P. Gendron et C. Faucher (2002). Les nouvelles stratégies de coaching. Les Éditions de l'homme. Ils ont également abordé la nécessité d'établir des liens entre les résultats attendus, les responsabilités et les compétences pour viser des actions pertinentes. Quant à la formulation de certaines compétences requises, elle est partiellement issue de la nomenclature de compétences de Michaël M. Lombardo et Robert W. Eichinger, les concepteurs de The Career Architect. Cet outil est notamment utilisé pour identifier les compétences de gestion dans plusieurs organisations. 